Please do not remove this page

RMIT

UNIVERSITY

\title{
Real-time trajectory optimisation models for next generation air traffic management systems
}

Gardi, Alessandro; Sabatini, Roberto; Ramasamy, Subramanian; Kistan, Trevor

https://researchrepository.rmit.edu.au/esploro/outputs/9921862165401341/filesAndLinks?institution=61RMIT_INST\&index=null

Gardi, A., Sabatini, R., Ramasamy, S., \& Kistan, T. (2014). Real-time trajectory optimisation models for next generation air traffic management systems. Applied Mechanics and Materials, 629, 327-332.

https://doi.org/10.4028/www.scientific.net/AMM.629.327

Document Version: Accepted Manuscript

Published Version: https://doi.org/10.4028/www.scientific.net/AMM.629.327

Repository homepage: https://researchrepository.rmit.edu.au

(C) 2014 Scientific.Net

Downloaded On 2023/04/26 13:06:04 +1000

Please do not remove this page 
Thank you for downloading this document from the RMIT Research Repository.

The RMIT Research Repository is an open access database showcasing the research outputs of RMIT University researchers.

RMIT Research Repository: http://researchbank.rmit.edu.au/

\section{Citation:}

Gardi, A, Sabatini, R, Ramasamy, S and Kistan, T 2014, 'Real-time trajectory optimisation models for next generation air traffic management systems', Applied Mechanics and Materials, vol. 629, pp. 327-332.

See this record in the RMIT Research Repository at:

https://researchbank.rmit.edu.au/view/rmit:24979

Version: Accepted Manuscript

Copyright Statement: (c) 2014 Scientific.Net

Link to Published Version:

http://dx.doi.org/10.4028/www.scientific.net/AMM.629.327 
This is the author pre-publication version. This paper does not include the changes arising from the revision, formatting and publishing process. The final paper that should be used for referencing is:

A. Gardi, R. Sabatini, S. Ramasamy and T. Kistan, "Real-Time Trajectory Optimisation Models for Next Generation Air Traffic Management Systems", Applied Mechanics and Materials, vol. 629, pp. 327-332, Trans Tech Publications, 2014. DOI: 10.4028/www.scientific.net/AMM.629.327

\title{
Real-Time Trajectory Optimisation Models for Next Generation Air Traffic Management Systems
}

\author{
Alessandro Gardi ${ }^{1}$, Roberto Sabatini ${ }^{1, a,{ }^{*}}$, Subramanian Ramasamy ${ }^{1}$ \\ and Trevor Kistan ${ }^{1}$ \\ ${ }^{1}$ School of Aerospace, Mechanical and Manufacturing Engineering, RMIT University \\ Melbourne, Victoria, Australia \\ aroberto.sabatini@rmit.edu.au
}

Keywords: 4-Dimensional Trajectory, Trajectory Optimisation, Air Traffic Management, Dynamic Airspace Management, Emissions Modelling.

\begin{abstract}
This paper presents models and algorithms for real-time 4-Dimensional Flight Trajectory (4DT) operations in next generation Communications, Navigation, Surveillance/Air Traffic Management (CNS/ATM) systems. In particular, the models are employed for multi-objective optimisation of 4DT intents in ground-based 4DT Planning, Negotiation and Validation (4-PNV) systems and in airborne Next Generation Flight Management Systems (NG-FMS). The assumed timeframe convention for offline and online air traffic operations is introduced and discussed. The adopted formulation of the multi-objective 4DT optimisation problem includes a number of environmental objectives and operational constraints. In particular, the paper describes a real-time multi-objective optimisation algorithm and the generalised expression of the cost function adopted for penalties associated with specific airspace volumes, accounting for weather models, condensation trails models and noise models.
\end{abstract}

\section{Introduction}

In order to progress towards a more sustainable aviation, it is of paramount importance to attain increasingly higher environmental and economic performances, evolving both the design, the operation and the auxiliary tasks, such as support and disposal, with a holistic approach. While the design of a single aircraft, of its components and of its reference mission can directly incorporate a significant number of scientific and technological outcomes, an essential consideration has to be given to its operation in the real traffic scenario. The Communication, Navigation, Surveillance and Air Traffic Management (CNS/ATM) and Avionics (CNS+A) domain is the essential link to ultimately enact the various improvements offered by new technologies and concepts in the operational context. It is envisaged that the adoption of 4-Dimensional Trajectory (4DT) concepts in conjunction with an increased use of automation and air-to-ground data-link communications in an Intent-Based Operations (IBO) context will provide the most significant benefits [1]. In this perspective, ongoing research has proposed the introduction of novel ground-based CNS/ATM systems that, in conjunction with airborne avionics, will enable IBO [2-5]. In line with this research path, we propose the introduction of a ground-based 4DT Planning, Negotiation and Validation (4PNV) system that, together with airborne Next-Generation Flight Management Systems (NG-FMS) enables optimised 4DT intent generation, negotiation and validation in real-time [6,7]. The optimisation of 4DT intents is one of the most promising and growing applications in the CNS $+\mathrm{A}$ context. Historically, flight planning was performed in a sort of pattern search between the available airways; subsequently, an optimal cruise level was calculated, and finally the vertical profiles for climb and descent were determined. As a matter of fact, although the optimisation of flight trajectories has been extensively studied, substantial potential benefits are left unattained due to the limited modelling of the system dynamics [8]. The inability to predictively handle the highly dynamic nature and uncertainties in real-time results in overall system inefficiencies. Therefore, the substantial dynamicity of the system has to be fully considered and implemented in all required IBO models and software tools. 
This is the author pre-publication version. This paper does not include the changes arising from the revision, formatting and publishing process. The final paper that should be used for referencing is:

A. Gardi, R. Sabatini, S. Ramasamy and T. Kistan, "Real-Time Trajectory Optimisation Models for Next Generation Air Traffic Management Systems", Applied Mechanics and Materials, vol. 629, pp. 327-332, Trans Tech Publications, 2014. DOI: 10.4028/www.scientific.net/AMM.629.327

\section{Operational Timeframes}

A large number of aspects in ATM are affected by uncertainties whose effects are integrated over time. Since the involved dynamics are often non-deterministic in nature, it is convenient to adopt a reference timeframe convention and formulate different optimisation problems, specifically tailored for each timeframe. The timeframe definition should be governed both by the numerical considerations and by operational motivations. From the numerical perspective, similarly governing dynamics and uncertainties of comparable magnitudes are requirements for a well-posed mathematical problem and hence good numerical performances. From the operational perspective, timeframe conventions that are already familiar or easily assimilated by the human operators are essential for the rapid and faultless deployment of new technologies. Based on these general principles, the first key distinction is between the online phase, when the air transportation mission is active, and the offline phase, when the mission is inactive. In the most recent terminology, ground operations form part of the online phase as well, as far as including payload boarding and disembarkation phases. In fact, variations in the ground timings are significantly propagated to the airborne phase. This heavy interdependence promote the formulation of a seamless gate-to-gate optimisation problem [9]. A subsequent timeframe distinction is based on the timespan before the occurrence of an unpredicted event affecting air traffic operations. Such unpredicted events would include any occurrence perturbing the successful outcome of the mission, such as potential conflicts, weather cells, airspace or airport congestions, and system failures. Barnier and Allignol's temporal classification of ATM operations provides a practical reference for this study, hence the adopted timespans are strategic, tactical and emergency [10]. Fig. 1 exemplifies the assumed timeframe definitions in line with our previous research $[6,7]$.

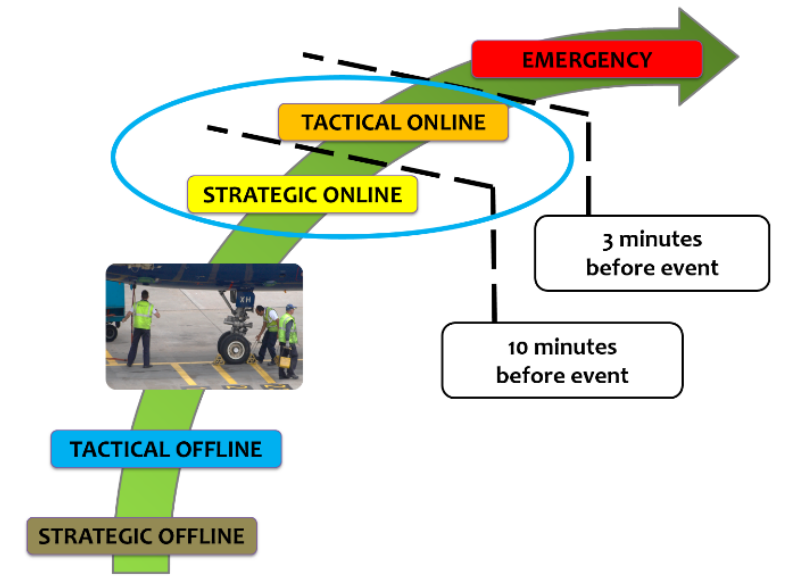

Figure 1. Assumed timeframes for the offline and online air traffic operations.

In the strategic timespan, the effect of uncertainties on a longer integration time causes a suboptimal estimation, but on the other hand the longer time available for calculation and decisionmaking allows the adoption of more complex models and algorithms in a higher dimensional optimisation problem. In the tactical timespan, uncertainties cause minor effects, but a safe solution to the problem shall be attained in a very limited time, thus the involved models and decision logics and the overall problem dimensions are necessarily simpler and typically based on approximations and linearisations. The emergency phase is traditionally associated with the adoption of robust and faultless decision logics for aircraft safety. The boundaries between strategic, tactical and emergency are usually set as part of the problem formulation. However, there remains considerable uncertainty as to the exact delineation between strategic online and tactical online air traffic operations, and a distinction based on the type of action to be taken is recently prevailing [11]. In particular, strategic online operations are mostly consisting of tactical Air Traffic Flow Management (ATFM) actions, initiated by a flow manager before the flight enters the jurisdiction who will have to implement the measures, whereas tactical online operations can be assumed to occur within the current sector and therefore already responsibility of the jurisdiction controller. 
This is the author pre-publication version. This paper does not include the changes arising from the revision, formatting and publishing process. The final paper that should be used for referencing is:

A. Gardi, R. Sabatini, S. Ramasamy and T. Kistan, "Real-Time Trajectory Optimisation Models for Next Generation Air Traffic Management Systems", Applied Mechanics and Materials, vol. 629, pp. 327-332, Trans Tech Publications, 2014. DOI: 10.4028/www.scientific.net/AMM.629.327

\section{Multi-Objective 4DT Optimisation Problem Formulation}

From an analytical perspective, the generalised trajectory optimisation problem can be expressed as $[12,13]$ : "Determine the states $\boldsymbol{x}(t) \in \mathbb{R}^{n}$, the controls $\boldsymbol{u}(t) \in \mathbb{R}^{m}$, the parameters $\boldsymbol{p} \in \mathbb{R}^{q}$, the initial time $t_{0} \in \mathbb{R}$ and the final time $t_{f} \in \mathbb{R} \mid t_{f}>t_{0}$, that optimise the performance indexes

$$
\boldsymbol{J}=\boldsymbol{\Phi}\left[\boldsymbol{x}\left(t_{0}\right), \boldsymbol{x}\left(t_{f}\right), \boldsymbol{p}\right]+\int_{t_{0}}^{t_{f}} \mathcal{L}[\boldsymbol{x}(t), \boldsymbol{u}(t), \boldsymbol{p}] d t
$$

subject to the dynamic constraints

$$
\dot{\boldsymbol{x}}(t)=\boldsymbol{f}[\boldsymbol{x}(t), \boldsymbol{u}(t), t, \boldsymbol{p}]
$$

to the path constraints

$$
\boldsymbol{C}_{\min } \leq \boldsymbol{C}[\boldsymbol{x}(t), \boldsymbol{u}(t), t ; \boldsymbol{p}] \leq \boldsymbol{C}_{\max }
$$

and to the boundary conditions

$$
\boldsymbol{\Phi}_{\min } \leq \boldsymbol{\Phi}\left[\mathbf{x}\left(\mathrm{t}_{0}\right), \mathbf{x}\left(\mathrm{t}_{f}\right), \mathbf{u}\left(\mathrm{t}_{0}\right), \mathbf{u}\left(\mathrm{t}_{f}\right) ; \mathbf{p}\right] \leq \boldsymbol{\Phi}_{\max }
$$

In order to introduce multi-objective optimality, it is necessary to adopt a technique for the predetermination of a unified performance index $J$, as in the a priori approach, or for the postprocessing of the results as in the a posteriori approach [14]. Since we are focussing on real-time optimisation algorithm, the weighted sum method, belonging to the category of a priori articulation of preferences, is adopted. The a priori approach is conceptually represented in Fig. 2.

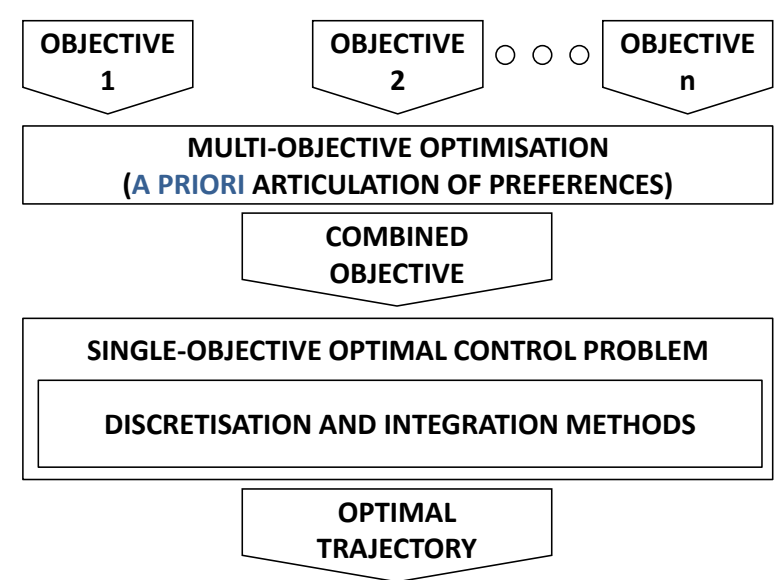

Figure 2. Block diagram of multi-objective optimisation with a priori articulation of preferences.

Gradient-based methods, typically associated with relatively quick computational performances, are usually employed to solve the Optimal Control Problem (OCP) in the a priori approach but their nonglobal convergence is a major drawback. The significant number of nonlinearities present in the models (aircraft dynamics, weather, etc.) give birth to multiple local minima in the cost functions. A promising solution lays in the adoption of a hybrid approach, with either a pattern search or an evolutionary algorithm performed as a first instance to determine the global convergence region, and subsequently a gradient based iteration to attain local optimality. Our current real-time 4DT optimisation algorithm is based on gradient-based iterations, as described in [6,7], and a hybrid extension is being implemented, by introducing a preliminary discrete pattern search. The multiobjective 4DT optimisation algorithm is conceptually represented in Fig. 3. All objectives and constraints shall be an integral part of the OCP formulation, in order to take advantage of the benefits guaranteed by the optimal control theory [13]. This last consideration substantially inhibits a pure black-box implementation. It is also useful to note that from a numerical perspective, it is convenient to supply the Jacobian matrix to the solver, whenever feasible, to prevent a complete recalculation at each iteration. 


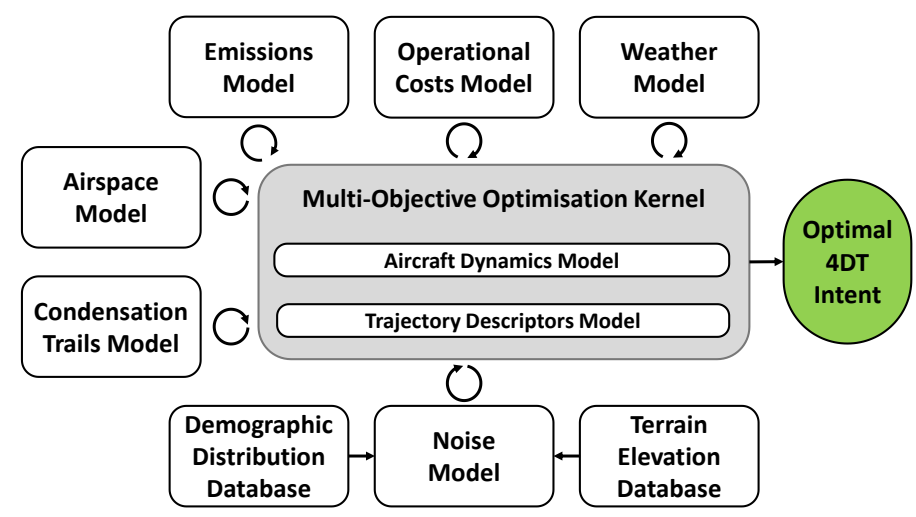

Figure 3. Block diagram of the multi-model 4DT optimisation algorithm.

In line with the described OCP formulation, we adopt the following generalised expression of the cost function in Eq. 1 for penalties associated with the transit through a dynamic penalty volume:

$$
J_{i}=\sum_{j=1}^{n_{\text {sect }}} \int\left[P F_{i, j} \cdot\left(\boldsymbol{x}(t) \cap S_{j}(t)\right)\right] d t
$$

where:

$J_{i} \quad=$ Performance/penalty index associated with the $i^{\text {th }}$ objective;

$P F_{i, j}=$ Penalty Factor associated to the $\mathrm{i}^{\text {th }}$ objective in the $\mathrm{j}^{\text {th }}$ penalty volume;

$\boldsymbol{x}(t)=$ Trajectory;

$S_{j}(t)=\mathrm{j}^{\text {th }}$ dynamic penalty volume.

Eq. 5 is used, in the case of 4-PNV and NG-FMS, with suitable forms of the Airspace Model (ASM), Condensation Trails Model (CTM), Weather State Model (WSM) and Noise Emission Model (NEM), by setting the associated penalty factors to adequate values. In our 4-PNV and NGFMS algorithm implementation, the computation of the portion of trajectory inside the penalty volume (Eq. 5) is supported by the Geospatial Data Abstraction Library (GDAL/OGR).

\section{Simulation and Results}

The described optimisation models were successfully employed in the proposed 4-PNV and NGFMS systems. The assessment of 4DT intents generation, negotiation and validation algorithms is performed in worst-case air traffic operations. In particular, Terminal Manoeuvring Area (TMA) peak-hour traffic is assumed. The TMA is modelled as an airspace volume of parallelepiped shape with a square base area of $60 \mathrm{~nm}$ per side, floor set at $2000 \mathrm{ft}$ and ceiling set at FL150. A single ILS approach procedure on a bearing of $160^{\circ}$ is introduced, transiting traffic at various inbound/outbound altitudes and exit points, and departing traffic towards a selected exit point. The focus of this simulation test case is on the arrival sequencing problem in perturbed conditions: the single landing runway available implies that not all traffics can be cleared to overfly the merge-point at their originally intended time. In order to be adopted for both strategic online and tactical online contexts, with a reference time horizon of 10 minutes, it is assumed that the total duration of optimization, negotiation and validation processes must remain under 300 seconds. Trajectories are checked for conflicts in the lateral, vertical and longitudinal directions. The 4-PNV identifies the best arrival sequence among the available options. Aircraft types included in the simulation belong to the Airbus A320 family, with 3 degrees of freedom aircraft dynamics and initial weight between $48000 \mathrm{~kg}$ and $60000 \mathrm{~kg}$. The optimal intents calculated by each NG-FMS consists of 4D waypoints, in a number variable between 3 and 30, towards the exit points or towards a merge point - the ILS Approach Fix (IAF). Two 4DT intents are calculated for each traffic: an optimal trajectory for minimum fuel and an optimal trajectory for minimum time. Avoidance of the urban Noise Sensitive Area (NSA) is granted by the inclusion of the NEM outputs and Awakening Noise Thresholds (ANT) in the 
This is the author pre-publication version. This paper does not include the changes arising from the revision, formatting and publishing process. The final paper that should be used for referencing is:

A. Gardi, R. Sabatini, S. Ramasamy and T. Kistan, "Real-Time Trajectory Optimisation Models for Next Generation Air Traffic Management Systems", Applied Mechanics and Materials, vol. 629, pp. 327-332, Trans Tech Publications, 2014. DOI: 10.4028/www.scientific.net/AMM.629.327

computation. The merge-point, in particular, marks the beginning of the common approach leg, where longitudinal separation is required to ensure successful separation upon landing, and to prevent separation infringements in the approach phase itself. The common approach leg has been modelled as a typical ILS approach profile, starting at 9.5 nautical miles from the runway at 3000 feet. The 4-PNV is capable of performing point-merge also further away from the approach, and on future steep final approach legs. After the initial intents have been stored in the 4-PNV, the pointmerge sequencing algorithm allocates the available time slots accordingly. The assumed minimum longitudinal separation is 4 nautical miles on the approach path for medium category aircraft approaching at 140 knots, therefore the generated time slots are characterized by a 90 seconds separation. Fig. 4 represents the results of one TMA point-merge simulation run.

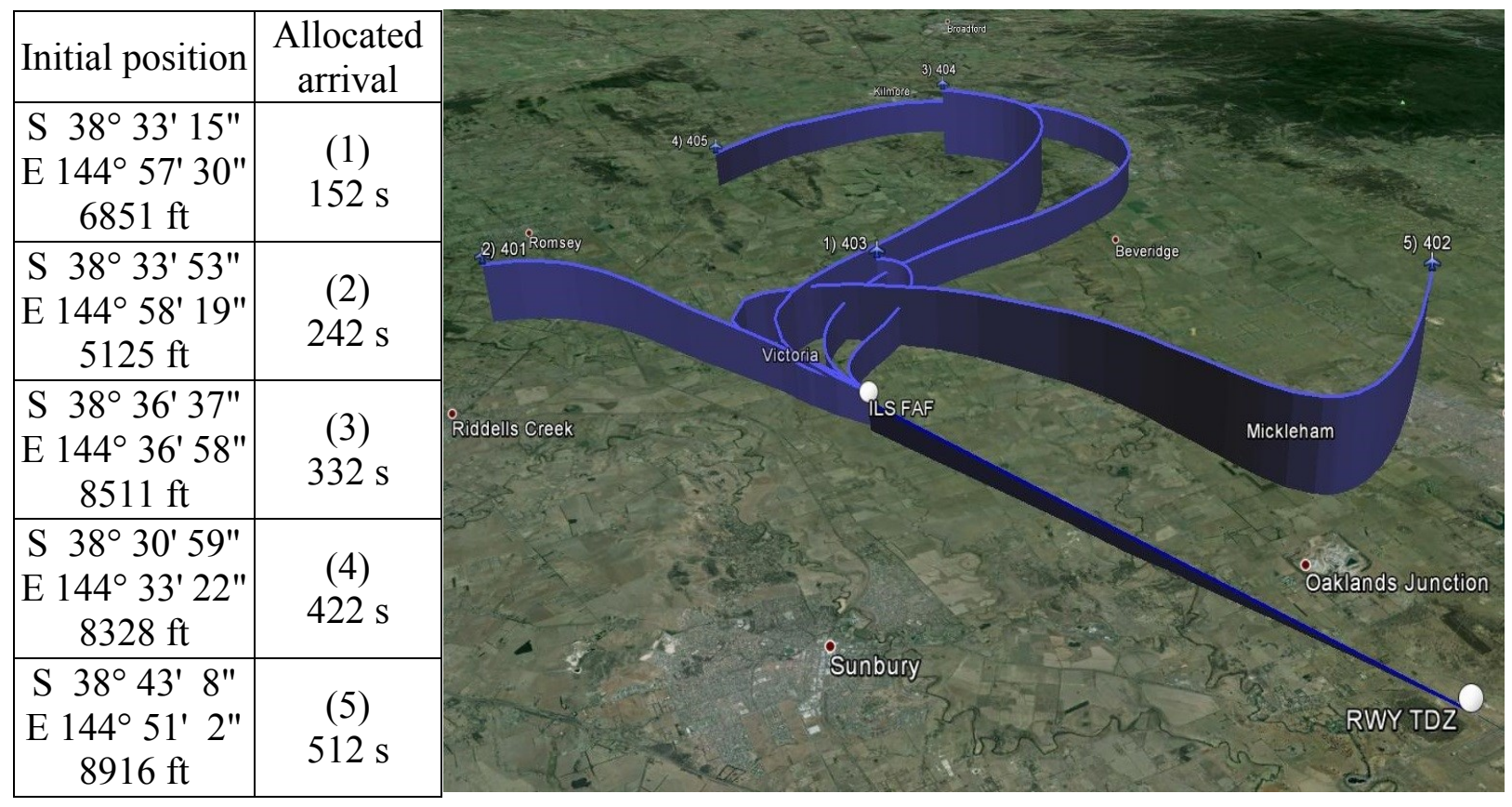

Figure 4. Results of 4DT intent generation, negotiation and validation in the TMA scenario.

\section{Conclusions and Future Work}

In this paper we presented the key features of the 4-Dimensional Trajectory (4DT) optimisation algorithms implemented in the CNS/ATM and Avionic (CNS+A) systems currently being researched, namely the ground-based 4-Dimensional Trajectory Planning, Negotiation and Validation (4-PNV) system and the airborne Next Generation Flight Management System (NGFMS) $[6,7]$. The assumed timeframe convention for offline and online ATM operations is presented and discussed in some details. The overall layout of the multi-objective optimisation algorithm is presented and discussed. A generalised expression for the cost functions associated with penalty volumes is described. This expression is used with suitable forms of the Airspace Model (ASM), Condensation Trails Model (CTM), Weather State Model (WSM) and Noise Emission Model (NEM). Future publications will address the detailed derivation of the state equations associated with environmental and economic performance models. Simulation of worst-case scenarios is performed for validation purposes. The proposed 4-PNV/NG-FMS systems are capable to meet the stringent real-time computational requirements for tactical online rerouting operations. Future research will assess the NG-FMS 4DT intent generation and execution performance in perturbed conditions, based on different navigation architectures and in combination with Avionics-Based Integrity Augmentation strategies [15, 16]. Novel sensing techniques for aviation related pollutants and atmospheric boundary layer/wake turbulence, currently being researched, will provide valuable inputs for dynamic air traffic optimisation $[17,18]$.

\section{References}

[1] SESAR Consortium, The ATM Target Concept - D3, SESAR Definition Phase, 2007. 
This is the author pre-publication version. This paper does not include the changes arising from the revision, formatting and publishing process. The final paper that should be used for referencing is:

A. Gardi, R. Sabatini, S. Ramasamy and T. Kistan, "Real-Time Trajectory Optimisation Models for Next Generation Air Traffic Management Systems", Applied Mechanics and Materials, vol. 629, pp. 327-332, Trans Tech Publications, 2014. DOI: 10.4028/www.scientific.net/AMM.629.327

[2] Eurocontrol, Trajectory Negotiation in a Multi-sector Environment, Programme for Harmonised ATM Research in Eurocontrol (PHARE), Bruxelles, Belgium, 1997.

[3] J. E. Robinson III and D. R. Isaacson, A concurrent sequencing and deconfliction algorithm for terminal area air traffic control, in proceedings of AIAA Guidance, Navigation and Control Conference 2000 (GNC2000), Denver, CO, USA, 2000. DOI: 10.2514/6.2000-4473

[4] H. Erzberger, The automated airspace concept, in proceedings of $4{ }^{\text {th }}$ USA/Europe Air Traffic Management Research and Development Seminar (ATM2001), Santa Fe, NM, USA, 2001

[5] E. Mueller, Experimental Evaluation of an Integrated Datalink and Automation-Based Strategic Trajectory Concept, in proceedings of AIAA Aviation Technology, Integration and Operations conference 2007 (ATIO2007), Belfast, Northern Ireland, 2007. DOI: 10.2514/6.2007-7777

[6] A. Gardi, R. Sabatini, S. Ramasamy, and K. de Ridder, 4-Dimensional Trajectory Negotiation and Validation System for the Next Generation Air Traffic Management, in proceedings of AIAA Guidance, Navigation, and Control Conference 2013 (GNC2013), Boston, MA, USA, 2013. DOI: $10.2514 / 6.2013-4893$

[7] S. Ramasamy, R. Sabatini, A. Gardi, and Y. Liu, Novel flight management system for real-time 4-dimensional trajectory based operations, in proceedings of AIAA Guidance, Navigation, and Control Conference 2013 (GNC2013), Boston, MA, USA, 2013. DOI: 10.2514/6.2013-4763

[8] Eurocontrol - SESAR JU, European ATM Master Plan - The Roadmap for Sustainable Air Traffic Management, 2nd ed, Brussels, Belgium, 2012.

[9] SESAR Joint Undertaking, SESAR and the Environment, Brussels, Belgium, 2010.

[10] N. Barnier and C. Allignol, Trajectory deconfliction with constraint programming, The Knowledge Engineering Review, vol. 27, pp. 291-307, 2012. DOI: 10.1017/s0269888912000227

[11] ICAO, Report of the Air Traffic Flow Management Seminar and Report of the Second Meeting of the Asia/Pacific ATFM Steering Group (ATFM/SG/2), Hong Kong, China, 2013.

[12] A. V. Rao, Trajectory Optimization, in Encyclopedia of Aerospace Engineering, Wiley, 2010.

[13] J. T. Betts, Practical methods for optimal control and estimation using nonlinear programming, $2^{\text {nd }}$ ed. vol. 19: SIAM, 2010.

[14] R. T. Marler and J. S. Arora, Survey of multi-objective optimization methods for engineering, Structural and Multidisciplinary Optimization, vol. 26, pp. 369-395, 2004. DOI: 10.1007/s00158003-0368-6

[15] R. Sabatini, T. Moore, and C. Hill, A new avionics-based GNSS integrity augmentation system: Part 1 - Fundamentals, Journal of Navigation, vol. 66, pp. 363-384, 2013. DOI: $10.1017 / \mathrm{S} 0373463313000027$

[16] R. Sabatini, T. Moore, and C. Hill, A new avionics-based GNSS integrity augmentation system: Part 2 - Integrity flags, Journal of Navigation, vol. 66, pp. 501-522, 2013. DOI: $10.1017 / \mathrm{S} 0373463313000143$

[17] A. Gardi and R. Sabatini, Unmanned aircraft bistatic lidar for $\mathrm{CO} 2$ colum density determination, in proceedings of IEEE Metrology for Aerospace Conference 2014, Benevento, Italy, 2014

[18] M. Marino, S. Watkins, R. Sabatini, and A. Gardi, Unsteady Pressure Measurements on a MAV Wing for the Design of a Turbulence Mitigation System, in proceedings of IEEE Metrology for Aerospace Conference 2014, Benevento, Italy, 2014 